\title{
Boolean Modeling of Biochemical Networks
}

\author{
Tomáš Helikar ${ }^{1}$, Naomi Kochi² ${ }^{2}$ John Konvalina ${ }^{3}$ and Jim A. Rogers*,1,3
}

\author{
${ }^{I}$ Department of Pathology and Microbiology, University of Nebraska Medical Center, 983135 Nebraska Medical \\ Center, Omaha, NE 68198, USA; ${ }^{2}$ Department of Genetics, Cell Biology, and Anatomy, University of Nebraska Medical \\ Center, 985805 Nebraska Medical Center, Omaha, NE 68198, USA; ${ }^{3}$ Department of Mathematics, University of \\ Nebraska at Omaha, 6001 Dodge Street, Omaha, NE 68182, USA
}

\begin{abstract}
The use of modeling to observe and analyze the mechanisms of complex biochemical network function is becoming an important methodological tool in the systems biology era. Number of different approaches to model these networks have been utilized-- they range from analysis of static connection graphs to dynamical models based on kinetic interaction data. Dynamical models have a distinct appeal in that they make it possible to observe these networks in action, but they also pose a distinct challenge in that they require detailed information describing how the individual components of these networks interact in living cells. Because this level of detail is generally not known, dynamic modeling requires simplifying assumptions in order to make it practical. In this review Boolean modeling will be discussed, a modeling method that depends on the simplifying assumption that all elements of a network exist only in one of two states.
\end{abstract}

Keywords: Systems biology, dynamical modeling, signal transduction, boolean networks.

The modern era of cellular biochemistry has been characterized by the discovery of large interaction networks that are notable for their highly nonlinear connection structures. Such structures complicate study since they are often quite difficult to reduce into linear subsets that function in isolation from the embedding network [1,2]. It is for this reason that the concept of "systems biology" has been developed and is now being applied to these complicated biochemical networks $[1,3,4]$.

One of the first steps taken toward applying a systems approach to understanding biochemical networks was to begin organizing the connection information determined in the laboratory by creating connection maps (wiring diagrams) that could then be analyzed using graph theory concepts. While these studies have been highly successful [5], the fact that cells are dynamical systems means the next major step in the study of these systems involves the creation of models that take into account how they move and function in time. Thus we are now in a new era of creating large-scale dynamical models of biochemical systems.

An obvious choice for the modeling of dynamical systems is to describe the system with a system of differential equations, and this approach has been used extensively to study protein and/or gene interactions in a variety of relatively small models (for examples, see [6-9]). One of the advantages of continuous modeling via differential equations is that one can potentially describe molecular interactions with high precision and in quantitative terms that correspond to realistic laboratory measurements. However, that precision tends to make them computationally unwieldy for the large scale models necessary for the systems approach. There

*Address correspondence to this author at the Department of Mathematics, University of Nebraska at Omaha, 6001 Dodge Street, Omaha, NE 68182, USA; Tel: 402 554-3110: Fax: 402 554-2975; E-mail: jrogers@unmc.edu is currently a great deal of work being done in order to simplify and streamline continuous models in order to make them more tractable for large-scale models, and there has been success (for example, see [10]).

The alternative to the continuous approach is discrete modeling. Discrete modeling is a qualitative approach that begins with the simplifying assumption that each node in a network can have only one of some set number of possible values at any particular time point. The most severe simplifying assumption for a discrete model is binary; each node can have only one of two values at any time, usually designated as ON or OFF. Binary (or Boolean) models are the simplest discrete model, and thus the most computationally efficient, but the severity of the simplification immediately raises the question of its validity; is it possible to make models that display the dynamical richness of these systems when all elements exist only in one of two states? The use of Boolean modeling of biochemical systems was introduced by Kauffman [11], who also demonstrated that Boolean models preserve the dynamical features of the original system $[12,13]$. Because from a historical perspective the expression of genes has often been thought of in terms of ON/OFF (e.g., expressed or not expressed), the employment of Boolean models seemed quite natural. It is then no surprise that many subsequent Boolean models of gene networks and their analysis have been developed [14-19]. However, what about the modeling of the even more complex modern networks that make up the cellular interactome? Can Boolean modeling be used to model these complex systems?

In this review, we will survey efforts at modeling biochemical networks with Boolean modeling methods, and see that not only is it possible to use them, but also that they can provide a useful complement to standard continuous modeling. 
A

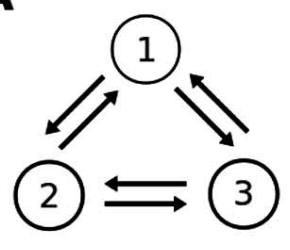

\begin{tabular}{lllll|lll|l}
2 & 3 & 1 & 1 & 3 & 2 & 1 & 2 & 3 \\
\hline 0 & 0 & 0 & 0 & 0 & 0 & 0 & 0 & 0 \\
0 & 1 & 0 & 0 & 1 & 1 & 0 & 1 & 1 \\
1 & 0 & 0 & 1 & 0 & 1 & 1 & 0 & 1 \\
1 & 1 & 1 & 1 & 1 & 1 & 1 & 1 & 1 \\
\multicolumn{1}{c}{ AND } & \multicolumn{3}{c}{ OR } & & \multicolumn{3}{c}{ OR }
\end{tabular}

$\mathrm{T} \quad \mathrm{T}+1$

\begin{tabular}{lll|lll}
1 & 2 & 3 & 1 & 2 & 3 \\
\hline 0 & 0 & 0 & 0 & 0 & 0 \\
0 & 0 & 1 & 0 & 1 & 0 \\
0 & 1 & 0 & 0 & 0 & 1 \\
0 & 1 & 1 & 1 & 1 & 1 \\
1 & 0 & 0 & 0 & 1 & 1 \\
1 & 0 & 1 & 0 & 1 & 1 \\
1 & 1 & 0 & 0 & 1 & 1 \\
1 & 1 & 1 & 1 & 1 & 1
\end{tabular}

B
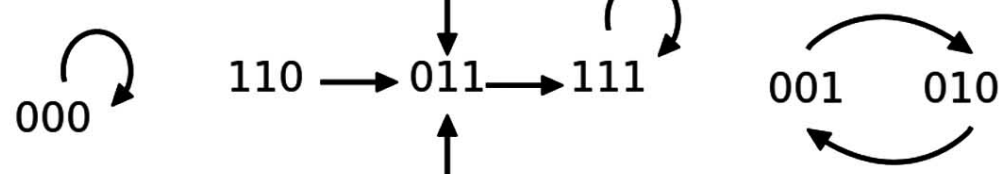

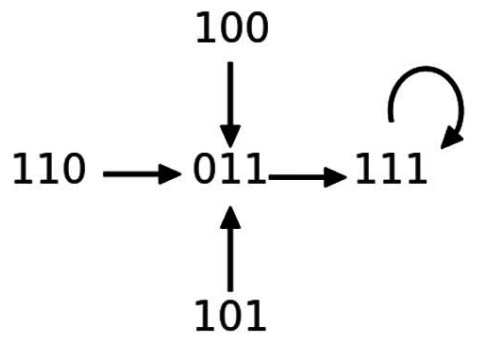

Fig. (1). (A) A simple network and its logical connections. (B) All possible trajectories and attractors for the network.

\section{THE ANATOMY OF NK BOOLEAN NETWORKS}

Detailed reviews of the concepts of Boolean modeling have been previously presented [20-22], but a simple introduction to Boolean models is presented here. Consider the simple network shown in Fig. (1A) [23]. There are three elements in this network and each element is connected to each of the others. Therefore, the parameter $N$ (the number of elements in the network) is 3 and the parameter $K$ (the number of connections feeding into each element) is 2 . The logical functions for each individual node, shown in the adjacent tables, are OR and AND. These tables give the ON/OFF state of each element (indicated with a 1 or 0 , respectively) as a function of the ON/OFF state of the other two elements connecting to it. The internal homogeneity parameter $P$ depends on how each Boolean function assigns 1 's and 0 's. $P$ is simply the percentage of times a function assigns 1 or 0 , whichever is greater. Thus for all three elements, $P$ is 0.75 since 1 is assigned $3 / 4$ 's of the time for nodes 2 and 3, while 0 is assigned 3/4's of the time for element 1 . Any function that assigns 1 (or 0 ) in $50 \%$ of the cases would have a value of 0.5 , the lowest possible value for $P$ (1.0 is the highest possible value). In the case of the network in Fig. (1), the average value of $P$ for the entire network is 0.75 , the average of the bias of all three elements.

The network exists at time $\mathrm{T}$ in some initial state, with each separate element either ON or OFF. At the next time ( $\mathrm{T}$ +1 ), the states of all three elements will change according to the tables shown. The evaluation of the entire system from time $\mathrm{T}$ to time $\mathrm{T}+1$ can be represented in a single table (shown on the right of Fig. 1A) where the column T contains all the possible initial states of the system and column $T+1$ shows the result of application of the logic set to each initial condition. Continued iteration by the same method results in a trajectory of the system as the states change over time. The trajectory that a given initial condition follows depends on all of the variables and parameters described above, and variation of the parameters can radically alter the types of trajectories obtained as will be discussed in the next section.

The network introduced in Fig. (1) is simple enough to view all of the possible trajectories, which are shown in Panel B. Because there are a finite number of elements in the system $(N)$, there is a finite number $\left(2^{N}\right)$ of possible states of the system. Thus, as the system travels in time, it must (regardless of trajectory) reenter a state previously encountered. As shown in Fig. (1B), when the system is at state 000 or 111, it remains there (encountering itself over and over), thus those two states are referred to as steady-states. If the system is at state 001 or 010 , it cycles between those two states, a trajectory that is referred to as a period 2 cycle. Finally, there are four other states of the system $(110,100,011$, and 101) that follow trajectories to the steady-state 111. In summary, the network described in Fig. (1) has three conditions (namely the two steady-states 000 and 111, and a period 2 cycle containing 001 and 010) called attractors into which the trajectories of all initial states eventually settle. In biological contexts, attractors of biochemical systems are often associated with cellular phenotypes [23-25].

Basins of attraction are those states whose trajectories lead to a given attractor. For example, the basin of attraction for the steady-state attractor 000 consists only of the condition 000. The basin of attraction for the steady-state attractor 111 is larger, consisting of $110,100,011$, and 101 . The basin for the period 2 cycle consists only of its two states, 001 and 010. Attractors are considered stable if they are resistant to minimal perturbation (defined as a change in a single element in the system [23]). One can easily see from Fig. (1B) that the steady-state 000 is not stable as a change in any single element results in the system moving to another attractor. For example, if 000 is changed to 100 , then the system is in a 
different basin that drains to a different attractor, namely 111. In contrast, the steady-state 111 is stable since any single change results in a state that is still in the same basin of attraction and will ultimately return to 111 .

\section{ORDER, CHAOS, AND COMPLEXITY IN NK BOOLEAN NETWORKS}

Boolean networks can have a wide variety of dynamics. The network considered in Fig. (1) has $N=3$ components and therefore $2^{N}=8$ possible states. Thus, the largest possible attractor would have size 8 since it is impossible to go more than 8 iterations without encountering a previous state. When networks have attractors that are large (relative to the size of the network), they are considered to be minimally ordered [23]. In other words, they are structured in such a way as to have a minimal number of reencounters with previous states. Furthermore, any network with such a structure would have attractors whose sizes scaled exponentially (by a factor of $2^{N}$ ) as the number of components in the system $(N)$ is increased [23]. Thus a minimally ordered system with $N=$ 200 components could have an attractor of size $2^{200}$, or approximately $1.6 \times 10^{60}$ ! An attractor of this magnitude means that the system would never repeat itself in any relevant time scale and could be considered effectively infinite. By definition, a trajectory of this type that is deterministic (and bounded) but not periodic over (effectively) infinite iteration is called chaotic [26]. As explained fully in [23], chaotic networks are characterized by high connectivity $(K>4)$ and low internal homogeneity of the Boolean functions $(P$ closer to 0.5 ). Thus networks with high values of $K$ and low values of $P$ can generally be characterized as chaotic. Networks with lower values for $K$ or higher values for $P$ tend to have smaller attractors that scale in size by a factor that is less than exponential and are characterized as ordered. Interest-
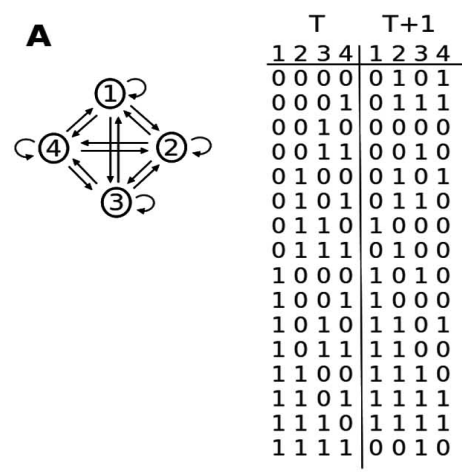

$\mathbf{B}$

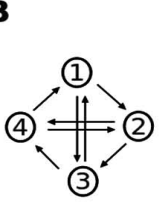

ingly, it has been shown that order in random $N K$ Boolean networks begins to crystallize automatically (i.e., attractors grow at a smaller rate as elements are added to the network) as $K$ approaches 2 or as $P$ approaches 1.0, and becomes a fully ordered system at $K=1$ [23]. Why would particular values of these two parameters alone determine order? The answer appears to be that networks with those parameter values contain a "frozen core" of elements, each frozen in either the 1 or 0 state. This can be seen by comparing two simple networks that differ only in those parameters.

Consider the network shown in Fig. (2A). Here we have $N=4, K=4$, and randomly assigned Boolean functions (average $P=0.53$ ); parameter values consistent with a relatively chaotic network. There are no steady-states, and all other trajectories feed into the period 8 cycle 0000, 0101, 0110, $1000,1010,1101,1111,0010$. In that period 8 cycle, all of the elements are in a perpetual state of change between 0 and 1. Although a period 8 attractor is not large in absolute terms, it is large relative to the size of the network $(N=4)$ and would grow as elements are added [23]. The fact that all trajectories lead to a relatively large cyclic attractor in which all of the elements of the network are in a state of flux is typical of a network that is chaotic [23]. This instability of elements also means that if any element is flipped to the opposite value, the system will generally end up on a very different place on the attractor. In large systems, that means that, even though on the same attractor, the ON/OFF pattern of each node will be very different than if the value had not been flipped. This is a type of "sensitivity to initial conditions," another trait that defines a chaotic system [26].

Contrast that with the network shown in Fig. (2B). In this case, $N=4, K=2$, and $P=0.75$; parameter values more in line with an ordered system [23]. All trajectories (except for the steady-state 0000) move to the steady-state 1111 where
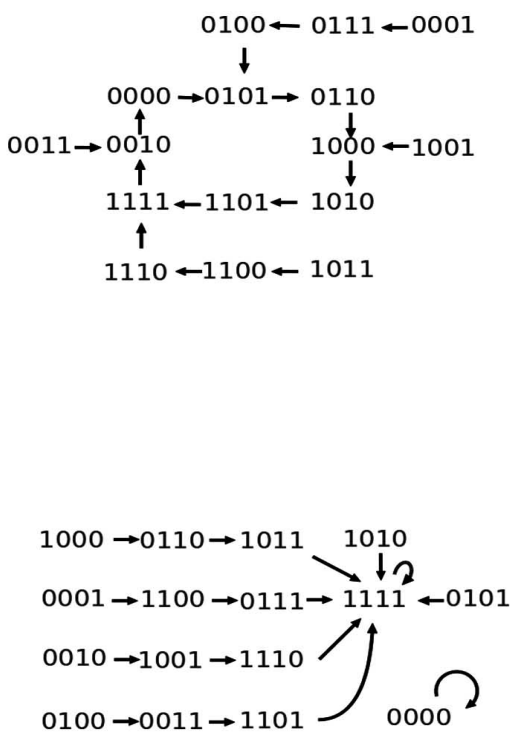

Fig. (2). (A) An $\mathrm{N}=4, \mathrm{~K}=4$ network with random Boolean functions (average $\mathrm{P}=0.53$ ). (B) $\mathrm{An} \mathrm{N}=4, \mathrm{~K}=2$, and $\mathrm{P}=0.75$ network with all $\mathrm{OR}$ connections. 
they become trapped forever. Hence all elements of the system eventually become frozen in the 1 position - a large frozen core of elements. Note that the frozen core is also stable as a single change of any element from 1 to 0 results in a state that is still in the basin of attraction for 1111; thus there is no sensitivity to initial conditions. The fact that all trajectories in this network lead to a small, stable attractor in which most (in this case all) of the elements of the network exist in a frozen core is typical of a network that is ordered [23].

Thus the parameters $K$ and $P$ are as indicators of relative order or chaos. However, there is another important Boolean function characteristic that influences the dynamics of a network; the number of functions with canalizing inputs [23]. Canalizing functions are Boolean functions that include at least one input variable that can determine the output regardless of the values of the other variables. Consider the Boolean function OR shown in Fig. (3A). Here variable $z$ has two input variables $x$ and $y$, and the state of variable $z$ is determined by the states of these two input variables according to the table. If the value (state) of $x$ is 1 , then the value of $z$ is 1 regardless of the value of $y$. In other words, the input variable $x$ set to the value 1 forces the value of $z$ to be 1 . The same is true for the input $y$. Thus, the Boolean function OR is a canalizing function because it has at least one canalizing variable (and in this case there are actually two). However, the Boolean function XOR shown in Fig. (3B) is not canalizing because setting each of the input variables to 0 or 1 does not "force" the state of variable $z$ to be constant.

Because of the "forcing" nature of canalizing functions, their presence in networks is associated with order. Exact formulas for the number of canalizing functions have been

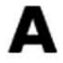

OR

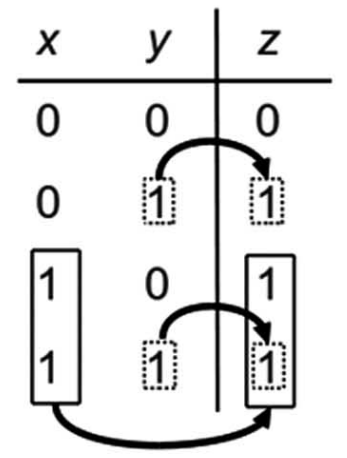

$$
\begin{aligned}
& x=1 \longrightarrow z=1 \\
& y=1 \longrightarrow z=1
\end{aligned}
$$

obtained [27]; if the number of input variables $K$ is greater than 4 , then there are relatively few canalizing functions, a fact consistent with highly connected networks tendency toward chaotic dynamics. Interestingly, biological networks are dominated by canalizing (forcing) functions [28]. Thus, the connectivity in biological networks can be greater than two, but with a correspondingly high number of canalizing functions, there can still be highly ordered dynamics typical of living systems [23]. Note that these conclusions are drawn from results from Kauffman $N K$ networks where the connectivity $K$ is fixed (a uniform degree distribution). The degree distributions of real biological networks tend to be power law distributions with exponents less than 2 [29], and there is evidence that the mean probability of canalizing functions for some biological systems is an effective indicator of the degree of order or stability in the system irrespective of the connectivity distribution [30].

However, living systems are not completely ordered. Because they must exhibit both stability (order) and flexibility (the ability to depart, at least temporarily, from strict order), they must have a degree of disorder. In fact, this is a third type of dynamic; complexity. A complex dynamic is defined as poised on the border between order and chaos, i.e., a system that is not fully ordered but not disordered to the point of being chaotic [23]. One can see that the network in Fig. (2A) is relatively chaotic but, as $K$ and $P$ are varied, the system becomes absolutely frozen as in Fig. (2B). Somewhere between those two extremes is the realm of complex systems, and the intermediate values of the parameters $K$ and $P$ can be considered definitive of a complex system. Complex Boolean networks tend to have moderately sized attractors with some, but not all, of the elements frozen while on the attractors. Complex dynamics is associated with

B

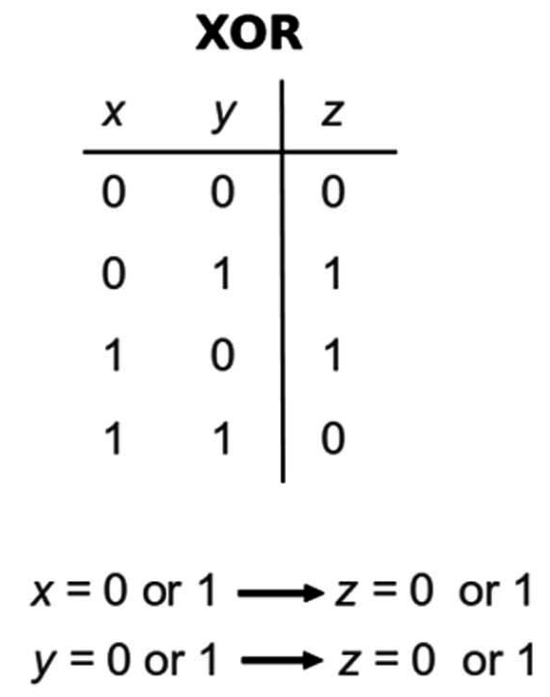

\section{$X$ is a canalizing variable. $y$ is a canalizing variable. $O R$ is a canalizing function.}

Fig. (3). (A) Both inputs are canalizing for the inclusive or (OR) function. (B) Neither input is canalizing for the exclusive or (XOR) function. 
living systems as they capture the balance between stability and flexibility that is a hallmark of living systems [23].

The balance between order and chaos necessary for nontrivial dynamics such as those demonstrated by living systems is an intriguing area of study, and Boolean networks have been applied in this area as well. By using a combinatorial approach Konvalina discovered a fractal component to nontrivial network dynamics in Boolean networks at $K=2$ [31]. This fractal, shown in Fig. (4), can be thought of as a visualization of the ratio of "freedom" and "control" in these networks. In this context, freedom and control means how much freedom there is to create networks (with arbitrarily large $N$ ) with particular behaviors (i.e., attractors). In other words, if a network with a particular behavior can be created in many different ways using different choices for the Boolean functions for the nodes that make it up, then that network is said to have a great deal of freedom. If, on the other hand, the creation of a network with that behavior requires careful selection from a limited set of choices for those functions, then there is a great deal of control in the network. It is intuitively evident that more connectivity in a network affords more choices as to how a network can be set up to produce a given dynamic, whereas lower connectivity limits those choices. Thus there is a fundamental relationship between connectivity and freedom/control in Boolean networks. Furthermore, in many complex systems the competing forces of freedom and control tend to shape the dynamics of those systems-- too much freedom in a system can lead to chaotic, disordered behavior, while too much control can result in totally ordered dynamics. Thus the discovery of the fractal represents a way to connect these dynamical properties (in a visual way) to the connectivity of the network. When $K=2$, the fractal representation shows a balance between freedom and control in $N K$ Boolean networks. On the other hand, lower values of $K$ indicate a bias toward control, while higher values of $K$ are shown as having a bias toward freedom. Thus the fractal shows that, at $K=2$, a phase transition between order and chaos occurs for the average $N K$ Boolean network, strongly supporting the concept that connectivity is a critical factor in the potential of networks to display the nontrivial behaviors associated with living systems.

With these basic concepts of states, attractors, and stability in mind, we can now see how Boolean models have proved useful in modeling biochemical systems at several levels, from basic network motifs such as feedback loops, to emergent behavior such as cellular information processing.

\section{MECHANISMS OF DYNAMICS; BOOLEAN STUDIES OF THE FEEDBACK LOOP}

One of the structural features commonly found in gene networks is the regulatory feedback loop. Feedback loops are network structures in which the output signal regulates the input signal of a given pathway. The direction of this regulation can be either positive or negative, hence the terms positive feedback loops or negative feedback loops. Biological feedback loops have been studied extensively [32, 33]. In biological systems, the existence of negative feedback loops in signaling networks gives rise to higher order, non-trivial properties such as adaptability and desensitization [34-37]. The mechanistic basis for this likely lies in the fact that negative feedback loops have been conjectured (and subsequently shown) to be essential for periodic behavior-- that is, they tend to keep a system on periodic attractors [32, 34, 38]. While this result has been confirmed in Boolean networks [39-42], Boolean modeling has also been used recently to show that as the number of feedback loops (particularly independent negative ones) increases in a network, the cyclic attractors of a network tend to become longer and the dynamics are much closer to chaotic [43]. Using a novel measure of independent negative feedback loops called "distanceto-positive-feedback," it was shown that, as the number of independent negative feedback loops increases, there tends to be a smaller number of larger cycles; a cycle structure associated with chaotic dynamics.

Positive feedback loops, on the other hand, result in multistability, hysteresis, ultrasensitivity, irreversibility, and the ability to set the amplitude of a signal [6, 34, 37, 44]. Collectively, these non-trivial properties appear to provide the cell with the ability to react to extracellular environmental conditions and stimuli even when the stimuli has been removed and the environment changed. This function is a crucial property of memory, hence it can be argued that one of the main functions of positive feedback is to form the basis for cellular "memory modules" [32, 45, 46]. The dependence of multistability on positive feedback loops has also been demonstrated in Boolean models [38-41, 47].

While the necessary association of negative and positive feedback loops with periodicity and multistability, respectively, is at this point well-documented in both the continuous and discrete case, Boolean modeling has recently been used to show that the nontrivial converse of these associations is also true-- i.e., that negative and positive feedback loops are sufficient for these dynamics [47]. This is an intriguing result since it implies that the existence of feedback loops in the graph structure of a network can provide information on the existence of periodic cycles and fixed points. This is an important finding given that most realisticallysized biological networks have state spaces far too large to visualize such cycles.

In addition to feedback loops in isolation, it is often the case that negative and positive feedback loops are integrated to form coupled regulatory feedback systems, giving rise to even more sophisticated and robust cellular functions [4850]. Again, Boolean models have been used to great effect in studies of coupled feedback loops. Using random Boolean networks, a series of papers by Kwon et al. has shown that networks with more coupled positive feedback loops are associated with multistability (i.e., fixed point dynamics), while those with more coupled negative feedback loops are associated with cyclical behavior [39, 51-53]. Moreover, the existence of coupled feedback loops is associated with increased robustness of the network (i.e., more resistant to perturbation); a finding that would explain why coupled feedback loops are so common in biological networks. Further study by Kwon et al. [54] found that coupled feedback loops are associated with networks that exhibit "preferential attachment." Preferential attachment is when a network evolves in such a way that nodes preferentially attach to nodes with higher numbers of attachments with other nodes, which results in a so-called "scale-free" structure [55]. However, they also found that networks evolved to have coupled 


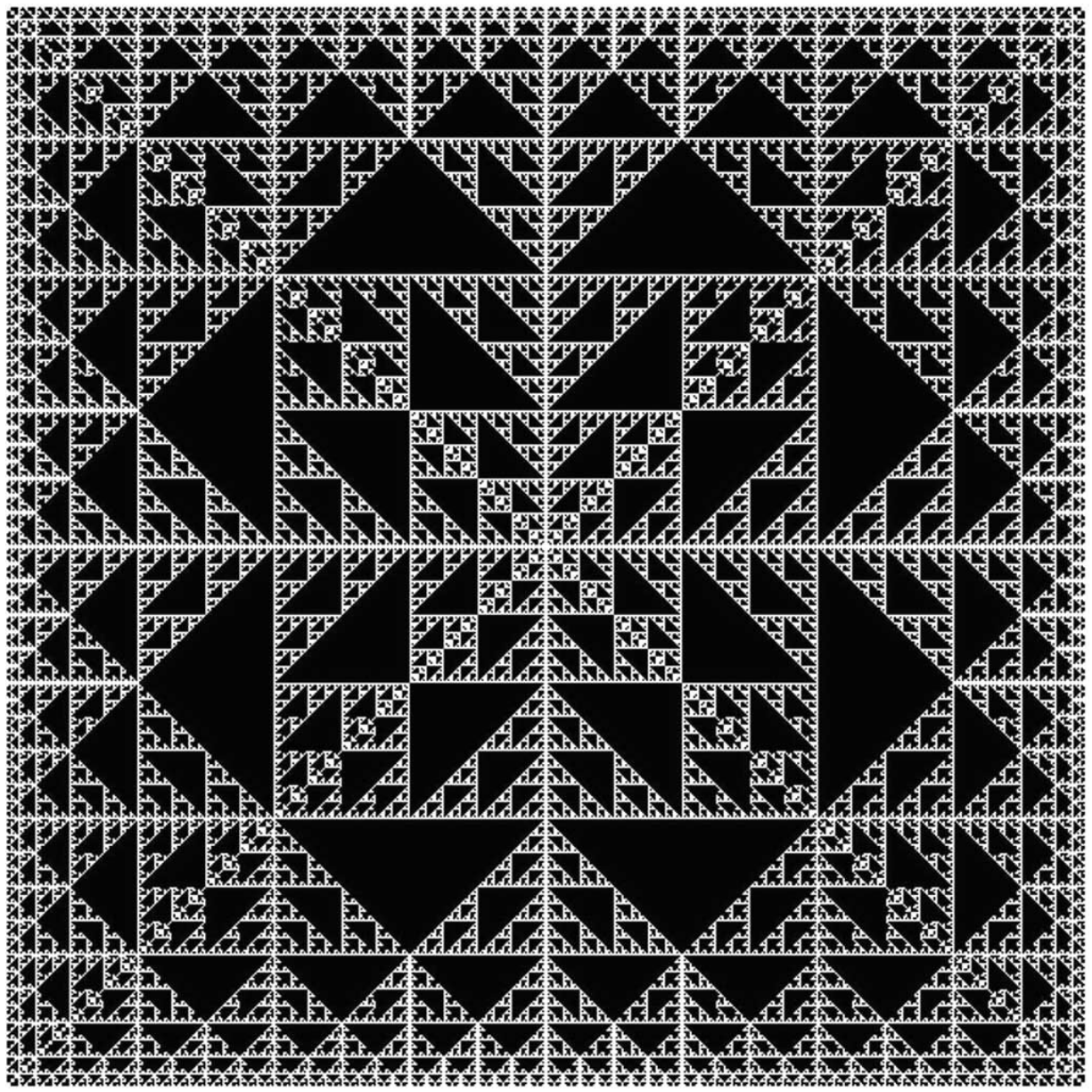

Fig. (4). The K2 fractal. As explained fully in [31], black areas represent trajectories of a Boolean network where all of the logic is fixed (i.e., control) whereas the white areas represent where there is choice in logic (i.e., freedom).

feedback loops are even more robust than preferential attachment networks, even though degree distributions are similar. This result was supported by the finding that older nodes in actual biochemical networks were associated with more coupled feedback loops. This is strong evidence that, while coupled feedback loops may have first appeared in biological systems spontaneously due to inherent preferential attachment, natural selection in favor of coupled feedback due to the superior robustness properties has resulted in their surprising prevalence in biochemical systems.

The feedback loop is one of the most significant network motifs in biochemical systems, and we have seen that Boolean modeling has been used extensively to understand the rich dynamics these motifs can produce. However, Boolean modeling has also been used extensively to understand specific biochemical mechanisms in cells.

\section{CELLULAR FUNCTION; BOOLEAN STUDIES OF BIOCHEMICAL MECHANISMS}

Because cellular signal transduction networks are so important for cellular function, these immensely complicated biochemical networks have been the subject of intense study for decades. However, the highly interconnected nature of signaling pathways means that the laboratory study of these networks is not always straightforward as the primary laboratory research tool of reductionism is not always effective [56]. Thus modeling has become an important component in biomedical research, and Boolean model is playing its part. Here is presented a survey of various results obtained using Boolean modeling.

In the area of research into mechanisms of apoptosis (programmed cell death), a number of Boolean models have been used. A Boolean model of caspase 3 and forkheadrelated transcription factor associated signaling pathways was used to develop a vulnerability assessment platform [57]. With this platform, the authors were able to identify proteins in the signaling network that may be more prone to pathologically important mutations, which may provide new insights as to identifying new targets for drugs, but more importantly, targets that may not be directly obvious unless a systems approach coupled with computational analysis is employed. In another study [58], a 40-node Boolean model of signal transduction network governing the processes of cellular survival and apoptosis was built to deepen the understanding of emergent properties such as stability and irreversibility that arise as a result of intricate architectural features of the system. These examples of a Boolean model of signal transduction in apoptosis was followed by a similar model of apoptosis developed by a different group [59] that used the model to identify feedback loops present in the system. This resulted in the identification of a previously unknown feedback loop, which may in turn lead to new studies as to the role of this feedback in the regulation of apoptosis. 
In the area of immunology, a Boolean model of over 50 signaling components in a cytotoxic T cell (CTL) was created to develop a better understanding of the survival of the cell in $\mathrm{T}$ cell large granular lymphocyte (T-LGL) leukemia [60]. Because the abnormal clonal expansion of TCL and their prolonged survival (or lack of apoptosis induction) is what characterizes T-LGL leukemia, the authors used the model to predict, for example, that when IL-15 and PDGF are constitutively activated, they are sufficient to induce other signaling abnormalities related to the disease.

Because of their relevance to disease (including breast cancer), a set of pathways of high interest to biomedical research include ones associate with the receptor tyrosine kinase (RTK) family [61-64]. This family comprises the EGFR (or ErbB1) as well as ErbB2, ErbB3, and ErbB4 [65], most of which have been considered an important target for cancer drug development [66, 67]. The activation of ErbB receptors is initiated upon the binding of a ligand (e.g., EGF, TGF-alpha, NRGs, etc.) to the receptor. The binding of a ligand to its receptor leads to homo- or hetero- dimerization, which provides the cell with the ability to amplify as well as diversify the signal [65, 68]. The improper regulation of these (and many downstream) signaling components, specifically the overexpression of some of EGFR and ErbB2, have been associated with cancer, namely breast cancer. Because of this realization, the ErbB components have been studied as potential targets for anticancer drugs. For example, the monoclonal antibody trastuzumab was developed to target ErbB2. However, in many cases cells develop resistance to the drug and hence create difficulties in the treatment. Therefore understanding the mechanisms of drug resistance and, especially identifying the cause of the resistance would have significant implications in the development of more efficient drug therapies. In fact, a recent study to analyze cell resistance to trastuzumab in relation to breast cancer was conducted by using a Boolean approach [69]. Specifically, a Boolean model of ErbB1-3 signaling as well as regulatory components of the G1/S transition of the cell cycle were synthesized. Using the computational systems approach, the signaling component c-MYC was identified as a potential novel target for drug therapies focus on breast cancer. In addition, the results obtained by the model predict interesting finding suggesting that therapeutic combinatorial targeting of ErbB receptors won't be effective in de novo trastuzumab resistant breast cancer cells.

In another study, a large-scale Boolean model of Epidermal Growth Factor Receptor (EGFR) and associated pathways was employed to study signaling dynamics in these pathways and compare the model's dynamics to highthroughput data [70]. The EGFR signaling model was developed by converting an existing stoichiometric model to logical terms that underlie Boolean models. Although the conversion process doesn't guarantee complete creation of all Boolean functions, a protocol was developed to overcome this shortcoming. Furthermore, a protocol to directly compare the dynamics of the Boolean model to high-throughput data was also developed. Comparing dynamics of the largescale Boolean models and the high-throughput data, the authors have identified several inconsistencies that may be attributed to various factors such as the model (in)completeness or cell type discrepancies (the Boolean model represents signaling system of all different types of cells, whereas the high-throughput data was obtained from human hepatocytes). However, being able to compare and map high-throughput data with the dynamics of Boolean models opens new doors to be able to identify gaps and/or problems in the large-scale models that can be subsequently expanded and/or fine-tuned. Having more complete models of complex signal transduction networks will provide investigators with a tool to perform additional in silico studies that will, in turn, lead to new predictions and generation of hypotheses that can be further tested in laboratory.

\section{EMERGENT PROPERTIES IN CELLS; BOOLEAN STUDIES OF GLOBAL CELLULAR DYNAMICS}

While modeling is useful for modeling specific mechanisms of biochemical function, the real promise of modeling lies in the development of a true systems approach to the study of the cell by modeling multiple biochemical networks simultaneously. Such large scale models have the potential to allow observation of how the cell integrates the multiplicity of inputs from the environment and generates appropriate responses. A major theme in the use of large-scale models is to explore the ability of the cell to map inputs (extracellular cues) to output (phenotypes; e.g., cellular fates).

The first example of this type of study was done focusing on the cell fate decision process using a Boolean model to abstract the underlying regulatory mechanism of cellular survival, apoptosis, and necrosis [71]. Results with this model demonstrated the ability to classify the output behavior into clear equivalence classes (corresponding to survival, apoptosis, and necrosis) based on the combination of the model inputs. Using this model, the authors were also able to generate new hypotheses and predictions as to the effects of different (virtual) knockouts on the decision making process. For instance, using computer simulations of their model, the authors were able to gain more insights into the role of RIP1 (a protein important in cell necrosis) in the cellular decisionmaking process. Specifically, it was found that RIP1 needs only to be transiently active for the cell's decision to undergo necrosis, whereas the protein was found to be constitutively active when cell survival was induced.

Another example of the use of a large-scale logical model to employ input-output mapping is work conducted in a Tcell environment [25]. The purpose of the study was to further understand the signaling process that leads to the activation and differentiation of primary T cells. A Boolean model of T-cell signaling system composed of 94 nodes and 123 connections was created by using qualitative information from the literature as well as the group's own experimental data. They employed existing methods applied in other modeling approaches (e.g., stoichiometric) of, for example, metabolic or gene regulatory systems, and demonstrated how these methods can be directly applied on qualitative models of signal transduction networks [72]. First, the large-scale model was analyzed from a graph-theoretical perspective by analyzing the model's interaction graph, and it was found that it contains the total of 172 feedback loops (89 negative and 83 positive), demonstrating their significance in cellular signaling systems. However, as mentioned in the introductory section of this review, although important, the analysis of static maps of signal transduction does not allow direct analysis of the dynamics of the system. Thus, the authors 
performed several analyses of the Boolean model to identify important cross-pathway dependencies, various input-output behaviors, and potential targets within the network that are capable of altering the global response of the signaling network to its external inputs. Collectively, these computational findings led to the generation of new hypotheses that the group tested experimentally. For example, the computational analysis of the model suggested the ability of active CD28 to induce and sustain the activity of the c-Jun N-terminal kinase (JNK), something that has not been experimentally established before. The authors subsequently confirmed this prediction in an in vitro experiment.

In the final example, Boolean modeling has been used to create a very large scale model in order to interrogate signal transduction networks for emergent properties, namely decision-making [24]. The weight of discoveries in recent decades has made it obvious that intracellular signaling pathways are not linear structures as first thought, but form highly complex non-linear structures. But why would this be the case? If the sole job of signaling pathways is to pass a signal from the outside of the cell to the nucleus, why the need for such a complexity in these structures? In many areas of cellular biochemistry, cells have evolved mechanisms that are elegant in their linearity and efficiency; the precise traits that made them susceptible to understanding by reductionist study decades ago. It seems incredible that the seemingly low-level function of simply passing a signal from the cell membrane to, for example, the nucleus would not have been solved by evolution with a similarly elegant and direct mechanism.

Approximately 20 years ago, a new hypothesis began to be developed that the function of signal transduction networks is more than a simple signal-passing; their role is much more sophisticated and complex -- it is to process information and make decisions based on the cell's environment [73]. In recent years evidence has grown that this revolutionary idea is indeed the case and, perhaps even more significantly, that this function is actually an emergent property of these complex networks; i.e., the overall functions of these networks cannot be observed or studied using reductionism.

To test the hypothesis that signal transduction networks function as an information processing, decision-making machine in the cell, a large-scale Boolean model of signal transduction in a generic fibroblast cell was constructed [24]. The model consists of several main signaling pathways, including the receptor tyrosine kinase (RTK), G-protein coupled receptor, and Integrin signaling pathway. The inclusion of these multiple pathways resulted in a model composed of about 130 Boolean nodes (representing the various signaling molecules) and over 800 connections. Via a novel technique in Boolean modeling, a stochastic aspect was introduced into the model which allowed simulation and interrogation of the model under tens of thousands of (random) virtual cellular environments. The simulation results were then analyzed using input-output mapping algorithms such as Principle Component Analysis to provide evidence that, indeed, there is a high likelihood that signal transduction networks have evolved to do more than simple signal-passing. In particular, the findings suggested that these networks, via their sensors (receptors), intake extracellular cues and, using their complex (and specific) architecture, process the information to produce clearly defined responses, even in the presence of noisy and often times contradictory environmental signals.

Building on these findings, in the second example, a 20+ node (stochastic) Boolean model integrating signaling pathways associated with Cadherin, Receptor Tyrosine Kinase, and Integrin/ECM signaling was used to study the global dynamics of that system [74]. These authors also used an input-output mapping approach. Specifically, they interrogated the model under all possible combinations of the external inputs (representing receptor ligands) and identified a set of attractors (corresponding to global cellular phenomena such as proliferation, apoptosis, etc.) in which the model's dynamics settled as a result of each input permutation. Creating an input-output map, the authors were also able to analyze the direct effects of some of the components on the output (phenotype) of the system. In addition, the model was analyzed while adding noise to the system. The results indicated that the system is very robust in that the input-output map was unchanged even under relatively high levels of noise. While the mechanism (and the modeled signaling pathways) used to introduce noise was different from that used in [24], the results suggesting a highly robust system are consistent.

\section{CONCLUSION}

Boolean models present a simplified alternative to the generally more common differential equation-based approaches. However, a reasonable objection is that the simplicity (e.g., binary states of the model's nodes) associated with the alternative discrete modeling techniques does not allow the capture of the richness of dynamics in the real biological systems. As we have seen, Boolean models have been applied to network dynamics studies at many different levels. In some of these studies, large-scale dynamical models were created (something that is often difficult using continuous modeling approaches) to reveal higher-order emergent properties of the signaling systems that would have been very difficult to perform using continuous modeling techniques.

However, there is an additional advantage to discrete modeling - the relatively simple mechanism of describing node interactions makes for an ideal platform for community-based model building. Because of the inherent size and complexity of biochemical networks, it is extremely difficult for a single person or group to efficiently transfer the vast amount of laboratory data into a mathematical representation; this fact applies to any modeling technique. One way to address this issue is to engage the community of laboratory scientists that have generated these data and, hence, have first-hand knowledge of the local protein-protein regulatory mechanisms. If the community of laboratory scientists had a mechanism by which they could collaborate by contributing their intimate knowledge of local interactions into a largescale global model, the creation of these models would be greatly enhanced in terms of both size and accuracy. Since Boolean models rely on qualitative information, it makes them an ideal candidate for that platform as most laboratory scientists communicate their data in qualitative terms.

Although the advantage of Boolean models for the creation of such a community-based modeling system is that they do not require an understanding of high level mathematics, it 
does assume that users dealing with these models are familiar with Boolean formalism. At first, this may seem as a subtle issue as most qualitative information generated in laboratories is practically generated and interpreted in Boolean terms (e.g., protein $x$ AND $y$ activate protein $z$ ), the Boolean truth table (and equations) get more complex as the size of the model increases. This complexity effectively creates another challenge in building large-scale models. However, this gap can be possibly bridged by software tools that would allow biologists to create Boolean models without having to directly interact with the model's mathematical complexities. Although, many of the existing tools offer numerous functions (e.g., GINsim [75], SQUAD [76], and ChemChains [77], Discrete Dynamics Labs [78], BooleanNet [79], BoolNet [80], or SimBoolNet [81]) and feature-rich graphical interfaces for modelers familiar with Boolean formalism, they still need much work before considered as "biologistfriendly."

However, as we move into the new era of dynamical modeling as an important complement to laboratory research, we have seen that Boolean modeling is a simple, understandable modeling method that has the power to represent very complicated systems. This combination of features has great appeal, and it is likely that Boolean modeling has earned its place alongside continuous modeling based on the results it will produce.

\section{REFERENCES}

[1] S. Bornholdt, "Systems biology. Less is more in modeling large genetic networks", Science, vol. 310, pp. 449-451, 2005.

[2] H. A. Kestler, C. Wawra, B. Kracher, and M. Kühl, "Network modeling of signal transduction: establishing the global view", Bioessays, vol. 30, pp. 1110-1125, 2008.

[3] H. Kitano, "Systems biology: a brief overview", Science, vol. 295, pp. 1662-1664, 2002.

[4] H. Kitano, "Computational systems biology", Nature, vol. 420, pp. 206-210, 2002.

[5] A. Ma'ayan, "Insights into the organization of biochemical regulatory networks using graph theory analyses", J. Biol. Chem., vol. 284, pp. 5451-5455, 2009.

[6] U. S. Bhalla, and R. Iyengar, "Emergent properties of networks of biological signaling pathways", Science, vol. 283, pp. 381-387, 1999.

[7] K. C. Chen, A. Csikasz-Nagy, B. Gyorffy, J. Val, B. Novak, and J. J. Tyson, "Kinetic analysis of a molecular model of the budding yeast cell cycle", Mol. Biol. Cell, vol. 11, pp. 369-391, 2000.

[8] B. Novák, and J. J. Tyson, "A model for restriction point control of the mammalian cell cycle", J. Theor. Biol., vol. 230, pp. 563-579, 2004.

[9] S. Legewie, N. Blüthgen, and H. Herzel, "Mathematical modeling identifies inhibitors of apoptosis as mediators of positive feedback and bistability", PLoS Comput. Biol., vol. 2, pp. e120, 2006.

[10] J. Feret, V. Danos, J. Krivine, R. Harmer, and W. Fontana, "Internal coarse-graining of molecular systems", Proc. Natl. Acad. Sci., USA, vol. 106, pp. 6453-6458, 2009.

[11] S. A. Kauffman, "Metabolic stability and epigenesis in randomly constructed genetic nets", J. Theor. Biol., vol. 22, pp. 437-467, 1969.

[12] L. Glass, and S. A. Kauffman, "Co-operative components, spatial localization and oscillatory cellular dynamics", J. Theor. Biol., vol. 34, pp. 219-237, 1972.

[13] L. Glass, and S. A. Kauffman, "The logical analysis of continuous, non-linear biochemical control networks", J. Theor. Biol., vol. 39, pp. 103-129, 1973.

[14] R. Thomas, "Boolean formalization of genetic control circuits", $J$. Theor. Biol., vol. 42, pp. 563-585,1973.

[15] R. Albert, and H. G. Othmer, "The topology of the regulatory interactions predicts the expression pattern of the segment polarity genes in Drosophila melanogaster", J. Theor. Biol., vol. 223, pp. 1$18,2003$.

[16] C. Espinosa-Soto, P. Padilla-Longoria, and E. R. Alvarez-Buylla, "A gene regulatory network model for cell-fate determination dur- ing Arabidopsis thaliana flower development that is robust and recovers experimental gene expression profiles", Plant Cell., vol. 16, pp. 2923-2939, 2004.

[17] S. Kauffman, "A proposal for using the ensemble approach to understand genetic regulatory networks", J. Theor. Biol., vol. 230, pp. 581-590, 2004.

[18] S. Nikolajewa, M. Friedel, and T. Wilhelm, "Boolean networks with biologically relevant rules show ordered behavior", Biosystems, vol. 90, pp. 40-47, 2007.

[19] M. Chaves, R. Albert, and E. D. Sontag, "Robustness and fragility of Boolean models for genetic regulatory networks", J. Theor. Biol., vol. 235, pp. 431-449, 2005.

[20] R. Thomas, "Laws for the dynamics of regulatory networks", Int. J. Dev. Biol., vol. 42, pp. 479-485,1998.

[21] S. Huang, "Genomics, complexity and drug discovery: insights from Boolean network models of cellular regulation", Pharmacogenomics, vol. 2, pp. 203-222, 2001.

[22] S. Bornholdt, "Boolean network models of cellular regulation: prospects and limitations", J. R. Soc.Interface., vol. 5, Suppl. 1, pp. S85-94, 2008.

[23] S. Kauffman, The origins of order, New York: Oxford University Press, 1993.

[24] T. Helikar, J. Konvalina, J. Heidel, and J. A. Rogers, "Emergent decision-making in biological signal transduction networks", Proc. Natl. Acad. Sci. USA, vol. 105, pp. 1913-1918, 2008.

[25] J. Saez-Rodriguez, L. Simeoni, J. A. Lindquist, R. Hemenway, U. Bommhardt, B. Arndt, U. Haus, R. Weismantel, E. D. Gilles, and S. Klamt, "A logical model provides insights into $\mathrm{T}$ cell receptor signaling", PLoS Comput. Biol., vol. 3, pp. e163, 2007.

[26] D. Kaplan, and L. Glass, Understanding nonlinear dynamics, New York, NY, USA: Springer-Verlag New York, Inc., 1995.

[27] W. Just, I. Shmulevich, and J. Konvalina, "The number and probability of canalizing functions", Physica. D., vol. 197, pp. 211-221, 2004.

[28] S. Harris, B. Sawhill, A. Wuensche, and S. Kauffman, "A model of transcriptional regulatory networks based on biases in the observed regulation rules", Complexity, vol. 7, pp. 23-40, 2002.

[29] F. Chung, L. Lu, T. G. Dewey, and D. J. Galas, "Duplication models for biological networks", J. Comput. Biol., vol. 10, pp. 677-687, 2003.

[30] F. Karlsson, and M. Hornquist, "Order or chaos in Boolean gene networks depends on the mean fraction of canalizing functions", Physica A Statistical Mechanics and its Applications, vol. 384, pp. 747-757, 2007.

[31] J. Konvalina, I. Konfisakhar, J. Heidel, and J. Rogers, "Combinatorial fractal geometry with a biological application", Fractals., vol. 14, pp. 133-142, 2006.

[32] O. Cinquin, and J. Demongeot, "Roles of positive and negative feedback in biological systems", C. R. Biol., vol. 325, pp. $1085-$ 1095, 2002.

[33] R. J. Prill, P. A. Iglesias, and A. Levchenko, "Dynamic properties of network motifs contribute to biological network organization", PLoS Bio.l, vol. 3, pp. e343, 2005.

[34] R. Thomas, D. Thieffry, and M. Kaufman, "Dynamical behaviour of biological regulatory networks--I. Biological role of feedback loops and practical use of the concept of the loopcharacteristic state", Bull. Math. Biol., vol. 57, pp. 247-276, 1995.

[35] N. Barkai, and S. Leibler, "Robustness in simple biochemical networks", Nature, vol. 387, pp. 913-917, 1997.

[36] T. M. Yi, Y. Huang, M. I. Simon, and J. Doyle, "Robust perfect adaptation in bacterial chemotaxis through integral feedback control", Proc. Natl. Acad. Sci. USA, vol. 97, pp. 4649-4653, 2000.

[37] C. P. Bagowski, J. Besser, C. R. Frey, and J. E. J. Ferrell, "The JNK cascade as a biochemical switch in mammalian cells: ultrasensitive and all-or-none responses", Curr. Biol., vol. 13, pp. 315-320, 2003.

[38] E. Remy, P. Ruet, and D. Thieffry, "Graphic Requirements for Multistability and Attractive Cycles in a Boolean Dynamical Framework", Adv. Appl. Math., vol. 41, pp. 335-350, 2008.

[39] Y. Kwon, and K. Cho, "Boolean dynamics of biological networks with multiple coupled feedback loops", Biophys. J., vol. 92, pp. 2975-2981, 2007.

[40] J. Aracena, J. Demongeot, and E. Goles, "On limit cycles of monotone functions with symmetric connection graph", Theor. Comput. Sci., vol. 322, pp. 237 - 244, 2004.

[41] E. Remy, and P. Ruet, "On differentiation and homeostatic behaviours of Boolean dynamical systems", In: Transactions on Computational Systems Biology VIII, Vol. 4780, Lecture Notes in Computer Science, Berlin, Heidelberg: Springer-Verlag, 2007, pp. 92101. 
[42] E. Remy, P. Ruet, and D. Thieffry, "Graphic requirements for multistability and attractive cycles in a Boolean dynamical framework", Adv. Appl. Math., vol. 41, pp. 335-350, 2008.

[43] E. Sontag, A. Veliz-Cuba, R. Laubenbacher, and A. S. Jarrah, "The effect of negative feedback loops on the dynamics of boolean networks", Biophys. J., vol. 95, pp. 518-526, 2008.

[44] C. P. Bagowski, and J. E. J. Ferrell, "Bistability in the JNK cascade", Curr. Biol., vol. 11, pp. 1176-1182, 2001.

[45] W. Xiong, and J. E. J. Ferrell, "A positive-feedback-based bistable 'memory module' that governs a cell fate decision", Nature, vol. 426, pp. 460-465, 2003.

[46] J. C. Sible, "Cell biology: thanks for the memory", Nature, vol. 426, pp. 392-393, 2003.

[47] E. Remy, and P. Ruet, "From minimal signed circuits to the dynamics of Boolean regulatory networks", Bioinformatics, vol. 24, pp. i220-6, 2008.

[48] T. Y. Tsai, Y. S. Choi, W. Ma, J. R. Pomerening, C. Tang, and J. E. J. Ferrell, "Robust, tunable biological oscillations from interlinked positive and negative feedback loops", Science, vol. 321, pp.126129, 2008.

[49] O. Brandman, and T. Meyer, "Feedback loops shape cellular signals in space and time", Science, vol. 322, pp. 390-395, 2008.

[50] O. Brandman, J. E. J. Ferrell, R. Li, and T. Meyer, "Interlinked fast and slow positive feedback loops drive reliable cell decisions", Science, vol. 310, pp. 496-498, 2005.

[51] Y. Kwon, and K. Cho, "Quantitative analysis of robustness and fragility in biological networks based on feedback dynamics", Bioinformatics, vol. 24, pp. 987-994, 2008.

[52] Y. Kwon, S. S. Choi, and K. Cho, "Investigations into the relationship between feedback loops and functional importance of a signal transduction network based on Boolean network modeling", BMC Bioinformatics, vol. 8, pp. 384, 2007.

[53] Y. Kwon, and K. Cho, "Coherent coupling of feedback loops: a design principle of cell signaling networks", Bioinformatics, vol. 24, pp. 1926-1932, 2008.

[54] Y. Kwon, and K. Cho, "Analysis of feedback loops and robustness in network evolution based on Boolean models", BMC Bioinformatics, vol. 8, pp. 430, 2007.

[55] A. Barabási, and Z. N. Oltvai, "Network biology: understanding the cell's functional organization", Nat. Rev. Genet., vol. 5, pp. 101$113,2004$.

[56] D. J. Wilkinson, "Stochastic modelling for quantitative description of heterogeneous biological systems", Nat. Rev. Genet., vol. 10, pp. 122-133, 2009.

[57] A. Abdi, M. B. Tahoori, and E. S. Emamian, "Fault diagnosis engineering of digital circuits can identify vulnerable molecules in complex cellular pathways", Sci. Signal., vol. 1, pp. ra10, 2008.

[58] Z. Mai, and H. Liu, "Boolean network-based analysis of the apoptosis network: Irreversible apoptosis and stable surviving", $J$. Theor. Biol., vol. 259, no. 4, pp. 760-769, 2009.

[59] R. Schlatter, K. Schmich, I. Avalos Vizcarra, P. Scheurich, T. Sauter, C. Borner, M. Ederer, I. Merfort, and O. Sawodny, "ON/OFF and beyond--a boolean model of apoptosis", PLoS Comput. Biol., vol. 5, p. e1000595, 2009.

[60] R. Zhang, M. V. Shah, J. Yang, S. B. Nyland, X. Liu, J. K. Yun, R. Albert, and T. P. J. Loughran, "Network model of survival signaling in large granular lymphocyte leukemia", Proc Natl. Acad. Sci. USA, vol. 105, pp. 16308-16313, 2008.

[61] D. F. Stern, "Tyrosine kinase signalling in breast cancer: ErbB family receptor tyrosine kinases", Breast Cancer Res., vol. 2, pp. 176-183, 2000.

[62] Y. Yarden, "Biology of HER2 and its importance in breast cancer", Oncology, vol. 61, (Suppl 2), pp. 1-13, 2001.

[63] T. Holbro, R. R. Beerli, F. Maurer, M. Koziczak, C. F. Barbas, 3rd and N. E. Hynes, "The ErbB2/ErbB3 heterodimer functions as an oncogenic unit: ErbB2 requires ErbB3 to drive breast tumor cell proliferation", Proc. Natl. Acad. Sci. USA, vol. 100, pp. 8933-8938, 2003.

[64] N. E. Hynes, and G. MacDonald, "ErbB receptors and signaling pathways in cancer", Curr. Opin. Cell. Biol., vol. 21, pp. 177-184, 2009.

[65] A. Citri, and Y. Yarden, "EGF-ERBB signalling: towards the systems level", Nat. Rev. Mol. Cell. Biol., vol. 7, pp. 505-516, 2006.

[66] M. A. Olayioye, R. M. Neve, H. A. Lane, and N. E. Hynes, "The ErbB signaling network: receptor heterodimerization in development and cancer", EMBO J., vol. 19, pp. 3159-3167, 2000.

[67] Y. Yarden, and M. X. Sliwkowski, "Untangling the ErbB signalling network", Nat. Rev. Mol. Cell. Biol., vol. 2, pp. 127-137, 2001.

[68] A. Zaczek, B. Brandt, and K. P. Bielawski, "The diverse signaling network of EGFR, HER2, HER3 and HER4 tyrosine kinase receptors and the consequences for therapeutic approaches", Histol. Histopathol., vol. 20, pp. 1005-1015, 2005.

[69] O. Sahin, H. Fröhlich, C. Löbke, U. Korf, S. Burmester, M. Majety, J. Mattern, I. Schupp, C. Chaouiya, and D. Thieffry, "Modeling ERBB receptor-regulated G1/S transition to find novel targets for de novo trastuzumab resistance", BMC Syst. Biol., vol. 3, p. 1, 2009.

[70] R. Samaga, J. Saez-Rodriguez, L. G. Alexopoulos, P. K. Sorger, and S. Klamt, "The logic of EGFR/ErbB signaling: theoretical properties and analysis of high-throughput data", PLoS Comput. Biol., vol. 5, p. e1000438, 2009.

[71] L. Calzone, L. Tournier, S. Fourquet, D. Thieffry, B. Zhivotovsky, E. Barillot, and A. Zinovyev, "Mathematical modelling of cell-fate decision in response to death receptor engagement", PLoS Comput. Biol., vol. 6, p. e1000702, 2010.

[72] S. Klamt, J. Saez-Rodriguez, J. A. Lindquist, L. Simeoni, and E. D. Gilles, "A methodology for the structural and functional analysis of signaling and regulatory networks", BMC Bioinformatics, vol. 7, p. $56,2006$.

[73] D. Bray, "Intracellular signalling as a parallel distributed process", J. Theor. Biol., vol. 143, pp. 215-231,1990.

[74] A. L. Bauer, T. L. Jackson, Y. Jiang, and T. Rohlf, "Receptor cross-talk in angiogenesis: mapping environmental cues to cell phenotype using a stochastic, Boolean signaling network model", $J$. Theor. Biol., vol. 264, pp. 838-846, 2010.

[75] A. G. Gonzalez, A. Naldi, L. Sánchez, D. Thieffry, and C. Chaouiya, "GINsim: a software suite for the qualitative modelling, simulation and analysis of regulatory networks", Biosystems, vol. 84, pp. 91-100, 2006.

[76] A. Di Cara, A. Garg, G. De Micheli, I. Xenarios, and L. Mendoza, "Dynamic simulation of regulatory networks using SQUAD", BMC Bioinformatics, vol. 8, p. 462, 2007.

[77] T. Helikar, and J. A. Rogers, "ChemChains: a platform for simulation and analysis of biochemical networks aimed to laboratory scientists", BMC Syst. Biol., vol. 3, p. 58, 2009.

[78] A. Wuensche, "Discrete dynamics lab: tools for investigating cellular automata and discrete dynamical networks," Artificial life models in software, In: Adamatzky A, Kosinski M, Eds.: Springer, London, 2009, pp. 215-258.

[79] I. Albert, J. Thakar, S. Li, R. Zhang, and R. Albert, "Boolean network simulations for life scientists", Source Code Biol. Med., vol. 3, p. 16, 2008.

[80] C. Müssel, M. Hopfensitz, and H. A. Kestler, "BoolNet--an R package for generation, reconstruction and analysis of Boolean networks", Bioinformatics, vol. 26, pp. 1378-1380, 2010.

[81] J. Zheng, D. Zhang, P. F. Przytycki, R. Zielinski, J. Capala, and T. M. Przytycka, "SimBoolNet-a Cytoscape plugin for dynamic simulation of signaling networks", Bioinformatics, vol. 26, pp. 141$142,2010$. 